\title{
The Idiosyncrasy of Beauty: Aesthetic Universals and the Diversity of Taste
}

\author{
Patrick Colm Hogan
}

\begin{abstract}
There are different senses of the word "beauty." It may refer to a broad social norm or to an individual psychological experience, what we might call "aesthetic response." The main contention of this chapter is that common or universal principles need not mandate nor even entail that everyone has the same experience of beauty. To the contrary, research indicates that the factors underlying aesthetic response predict considerable individual diversity. It initially seems that the search for universals of beauty is both hegemonic and falsely homogenizing. However, it is not hegemonic if we are concerned with aesthetic response, rather than social norms. Moreover, a clear understanding of universals does not preclude idiosyncrasy. In fact, when properly formulated, such universals predict and explain individual diversity.
\end{abstract}

Keywords Attachment $\bullet$ Beauty $\bullet$ Prototypes $\bullet$ Taste $・$ Aesthetic universals

\section{Aesthetic Universals: Some Initial Issues}

It seems that we have two choices in speaking about beauty. We can either find universal principles that define and explain what beauty is or we must set it aside as a coherent object of study. However, as soon as one mentions "universals" of beauty, one is faced with an obvious problem - the variability of taste. Professors and students of literature today are likely to phrase the problem in terms of culture, saying that cultures develop radically different conceptions of beauty. This presumption of internal cultural uniformity and external cultural difference is widespread today, but highly problematic on many grounds. (For a discussion of some of these problems, focusing on literature, see Hogan 2003; for a treatment of political problems with the culturalist view, see Eriksen and Stjernfelt 2012.) But one does not need to accept culturalist presuppositions to accept the main point.

P.C. Hogan $(\bowtie)$

Department of English and Program in Cognitive Science, University of Connecticut, Storrs, CT 06269-4025, USA

e-mail: patrick.hogan@uconn.edu 
Simply put, tastes differ. Even those of us who share the same general tastes do not always favor precisely the same works of art-and many of us do not share the same general tastes. To some extent, this is a matter of the ambiguous usage of such terms as "beautiful." But not all cases can be explained away so easily.

For example, confining ourselves to facial beauty-one of the areas where universality seems best established-Maxim Magazine recently proclaimed Miley Cyrus the most beautiful woman (http://news.yahoo.com/miley-cyrus-hottestwoman-world-photo-153229449.html). For me, she is perfectly fine, but nothing exceptional. In contrast, I recently saw an old film staring the Indian actress Tanuja, whom I found very lovely. But Tanuja has no particular reputation as a great beauty. The same point holds for men. One magazine proclaimed Josh Hartnett the world's most beautiful man (http://acidcow.com/pics/7706-top-100-most-beautiful-men100-pics.html). When I think of a good-looking man, someone like Raj Kapoor occurs to me, at least at a certain period. However, Kapoor is not renowned for his beauty.

In short, despite research indicating that test subjects judge attractiveness similarly across races (see Zebrowitz et al. 1993), it seems incontrovertible that individually we differ in our tastes. For many of my colleagues, this itself is definitive proof that there are no aesthetic universals. If beauty were a "universal value," then should we not all like Miley Cyrus (or Tanuja) and Josh Hartnett (or Raj Kapoor)? To make matters worse still, when I discuss aesthetic universals (e.g., in talks), I may be faced with the criticism that any universals are necessarily an imposition of oppressive standards-European, male, heterosexual, and so on.

The political objection is important. It is undoubtedly the case that people unselfconsciously generalize their own idiosyncratic aesthetic and other preferences. It is important to recognize and respond to this. In a political context, this may have an ideological function. Judgments of beauty may function to denigrate the artistic achievements of other cultures. In a political context, it is important to respond to that denigration practically, in terms of policies and social action. Moreover, in a scientific context, it is important to recognize the full range of aesthetic experience. In other words, the key scientific issue is the importance of non-dominant views as data. It is simply bad science to take, say, European male preferences-or rather the preference of European male professors of aesthetics - into account while ignoring everyone else. But this simply returns us to the issue of idiosyncrasy.

\section{Three Meanings of "Beauty"}

As a preliminary step in clarifying what is at stake in discussing principles of beauty, we may distinguish three different ways in which the term "beauty" is used. The first and simplest usage is what might be called "essential." This is the use of the term "beauty" whereby it refers to some property intrinsic to the object. A Platonist might say, for example, that there is a form or idea of beauty and all beautiful objects participate in that idea, even if the idea or, for that matter, the objects are unobserved. By this account, there is a fact as to whether Josh Hartnett is or is not beautiful, 
just as there is a fact as to whether the stuff in my cup is or is not water (i.e., $\mathrm{H}_{2} \mathrm{O}$ ). Not being a Platonist, I must admit that this conception of beauty-like other conceptions of objective essences - has no appeal for me. In fact, it seems ultimately a rather bizarre idea since it is not evident that we can relate this "objective" beauty to our subjective sense of beauty in any clear or systematic way. If the essential beauty is not linked with our subjective sense of beauty, it is not clear that we are speaking of the same thing in the two cases. However, if we are speaking of the same thing, then it is difficult to see how we could solve the problem of the diversity of taste. That diversity would seem to suggest that there is no essence or that, when tastes conflict, one person is right and another is wrong. In principle, the latter is possible. However, that leaves us with the problem of how to learn just what the objective essence is. That would seem to lead us into mysticism, which is (to say the least) scientifically problematic.

Another use of the word "beauty" is social and normative. This refers to what is accepted as beautiful in a given group. Knowing the cultural norms of beauty is part of social competence for participation in any group. (I use "cultural" very broadly to refer to the practices of any group - whether a large society or a small clique.) Thus, if I am among American English professors, I can assume that To the Lighthouse has high normative status. Personally, I vastly prefer Mrs. Dalloway (in fact, I prefer The Waves and Jacob's Room). Indeed, I do not actually care much for To the Lighthouse (I find Mr. Ramsay to be more of a cartoon than a character), while I find Mrs. Dalloway to be one of the greatest novels ever. Nonetheless, it is part of my social competence to know that, socially, To the Lighthouse has higher aesthetic status. To take a simpler example, extreme slenderness is part of the social norm for female beauty, while muscularity is a social norm for male beauty (see the discussion of body ideals in chapter three of Giles 2010). Since the word "beautiful" is commonly used to refer to the social norm, it makes sense to say, "She has a beautiful body, but I don't care for skinny women" or "He has a beautiful body, but I don't care for muscle-bound men." In contrast, it would be odd to say (of a plump woman), "She has a beautiful body, but I'd prefer her if she were slender" or (of a slender man), "He has a beautiful body, but I'd prefer if he had some muscle."

Colleagues who make political objections to aesthetic universals (e.g., "those on the political left for whom the aesthetic is simply 'bourgeois ideology", [Eagleton 1990 , p. 8]) probably have this socially normative view of beauty in mind. This is the area where political objections to "universalism" have their greatest force. For example, reflecting one segment of European norms, Thomas Babington Macaulay (1835) made a famous statement that "a single shelf of a good European library was worth the whole native literature of India and Arabia." This is the sort of statement that involves a specifically "hegemonic universalization" (as Lalita Pandit might put it [see Pandit 1995, p. 207]), an extension of one set of social norms to other societies. The same thing happens not only with nations, but also with classes and other groups. This is clearly problematic and an apt topic for political analysis and response.

On the other hand, none of this means that social norms are wholly outside the realm of universality. The issue is the level at which universality enters. Most 
obviously, social norms may differ in their particulars while remaining open to explanation in terms of universal principles. For example, there is a commonplace that the dominant views in a society are the views of the dominant class (e.g., this is implied by most uses of "hegemony" [see, for example, Williams 1985, p. 145 on the generality of hegemony]). Thus, bourgeois aesthetic norms are often seen as becoming dominant with the rise of capitalism; English aesthetic norms become dominant in English colonies, and so on. In some cases, the production of aesthetic norms may be understood as more complex and indirect, but still a matter of historical variations on dominant social conditions. For example, Watt argues that "The novel's serious concern with the daily lives of ordinary people" depends on "general conditions." The first of these is that "the society must value every individual highly enough to consider him the proper subject of its serious literature." This condition arises with "individualism," which is itself contingent on "the rise of modern industrial capitalism and the spread of Protestantism" (Watt 1957, p. 60). Other writers stress a different sort of complexity with resistant norms always playing a role, often in relation to politics. For example, having emphasized dominant discourse in Orientalism (Said 1978), Edward Said developed the topic of "cultural resistance" (Said 1993, p. xii) in Culture and Imperialism (see in particular chapter three). Susan Faludi has argued that social norms of female beauty, such as extreme thinness, have a role in sustaining patriarchy-and those norms change "during periods when the culture is more receptive to women's quest for independence" (1991, p. 204). In all these cases, we see general principles linking social norms of aesthetics to social domination and, for some, resistance to such domination. In each case, this is a form of universalism.

In the remainder of this essay, we will only give passing attention to social norms of beauty. Our focus will be on the third, the psychological sense of "beauty," what we might equally call "aesthetic response"-what one finds beautiful. Problems of idiosyncrasy arise most obviously for this psychological sense. If our psychological responses to beauty are different, how can there possibly be (psychological) aesthetic universals? However, our brief sketch of social norms in beauty has already indicated that variability need not be incompatible with universal principles, since the principles and the variability will occur at different levels-just as the law of universal gravitation and specific trajectories of gravitational motion occur at different levels in physics. We would not say that there is no universal gravitation because, in ordinary atmosphere, it takes longer for a feather to fall than a bowling ball. Here, as elsewhere, divergence in manifestation in no way contradicts universality in principles.

Indeed, one could go further in the case of psychological principles. When dealing with psychological response, we are almost necessarily dealing with a wide range of factors bearing on perception, memory, inference, emotion, and other systems and processes that are sometimes highly divergent from person to person. These all involve possible variability and their interaction only multiplies that variability. For instance, it seems to be the case that emotional response involves at least three factors: innate propensities, formative critical period experiences, and specific emotional memories (see chapter two of Hogan 2011b). The first 
will vary somewhat across individuals. The second will vary more significantly (e.g., there will be differences between secure and insecure attachment). Finally, specific emotional memories, though perhaps the least consequential, may vary considerably. Our experience of an artwork (e.g., a novel) clearly involves complex interactions of all components of emotional response, not to mention other variable cognitive processes. Indeed, the same point seems to hold even for our response to facial beauty.

Given the preceding points, it would be very surprising if universal principles predicted uniformity of aesthetic response. In fact, the nature of psychological processes predicts that universals of aesthetic response will lead to diversity in individual feeling. Put differently, given the complexity of the human mind, uniformity of response would seem to entail difference in principles, not uniformity of principles. To take a simple example from outside aesthetics, imagine that there is some life-threatening crisis. There are two mothers, Jane and Sally, each with her own child, baby Jane and baby Sally. Faced with the crisis, Jane tries to save baby Jane. If Sally tries to save baby Sally, then she is doing something different from Jane, but following the same general principle (roughly "Save your own child first"). In contrast, if she tries to save baby Jane, then she is engaging in the same manifest behavior as Jane (saving baby Jane), but it seems clear that she is not following the same principle. In short, far from being inconsistent with variability in taste, a cognitively based set of universal principles of taste would seem to predict such variability. In the following sections, we will consider just what variability it predicts.

\section{Aesthetic Response and Idiosyncrasy (I): Pattern Recognition and Endogenous Reward}

In earlier work, I have argued that empirical research on beauty suggests that aesthetic response involves a complex integration of cognitive and emotional factors (see 2013 and 2014). Specifically, there seem to be two key cognitive processes in aesthetic response and two key emotional systems. As to the first cognitive process, some research points to non-habituated pattern recognition as central to some aesthetic response, most obviously in music (see Vuust and Kringelbach 2010; this is presumably the principle operative in a range of other aesthetic experiences as well, ranging from decorative art to mathematics). Non-habituated means that we have not come to fully anticipate the pattern, but must discern it in the course of our experience. Or, rather, it may be that we do anticipate the pattern for background phenomena (e.g., a drum or bass sequence); however, we come to discern the pattern in foreground elements or phenomena that are the object of attentional focus (e.g., melodic variations) in the course of experience.

This cognitive aspect of aesthetic response appears to be connected with activation of the endogenous reward system (see Vuust and Kringelbach 2010, p. 266). 
The reward system governs seeking behavior and is related to our experience of pleasure (on reward "wanting" and reward "liking," see Fareri and Delgado 2013, p. 446). Like other emotional responses, the intensity of reward system activation is presumably connected with the gradient of change in the "eliciting conditions" for the emotion. In general, the intensity of a final emotion is affected by the change from a prior emotion. If, on being called to the Department Head's office, I anticipate being told that I won an award, I will respond differently to the announcement of the award than if I anticipated chastisement (cf. Ortony et al. 1988, p. 72 on disappointment and relief in relation to effort; the point seems generalizable to more passive conditions of anticipation). If, in aesthetic response, reward system activation is produced by pattern recognition, then one would expect the intensity of the response to be in part a function of the rapidity and extent of the change from disorientation to pattern recognition (as well as the effort involved, as Ortony et al. [1988] suggest). By "rapidity," I mean the timing. Intuitively, it seems that there is a certain temporal window in which pattern recognition has the right effect. A comparable case might be the understanding of a joke; it is only funny if one understands it immediately after the punch line, but usually not if one understands the pattern only after reflection on the joke. By "extent," I mean the degree of change from incomprehension to comprehension. Recurring to the example of a joke, it seems clear that our enjoyment of a witticism is diminished to the degree that we anticipate the punch line or anticipate features of that punch line.

We have here a very complex universal principle, or candidate for a universal principle, of aesthetic response. By this principle, aesthetic pleasure involves the following components (slightly elaborated beyond the foregoing): (1) a focal object and (often) a background object; (2) consistent pattern recognition for the background object; (3) non-habituation to the pattern in the focal object; (4) a shift from some degree of disorientation to fuller recognition of a pattern in the focal object within a certain temporal window; (5) reward system activation. There are undoubtedly other factors as well, for example, (6) limitation of the aversive quality of disorientation (as severe disorientation may lead to such aversive response that it inhibits subsequent pleasure at pattern recognition). The list here is not meant to be complete, but to indicate some of the complexity at issue. The key point for our present analysis is the following. This complex principle, with its multiple components, is a plausible, empirically supported candidate for a universal aesthetic principle. One may reasonably argue that an experience satisfying this principle gives rise to aesthetic enjoyment across cultures and historical periods. But it is clear that there will be a great deal of "output variation" in the application of these principles in particular circumstances. In other words, there will be considerable individual variation in aesthetic response.

We may consider the components in turn. First, there is the difference between a focal object and a background object. This would seem to be relatively uniform across individuals. However, it is not. Consider Hindustani classical music. A typical performance will include a drone playing the main notes of the piece, a drum, and a solo instrument or a vocalist. Even listeners unfamiliar with Hindustani music are likely to focus their attention on the solo instrument, allowing the drone 
and drum to provide a patterned frame that aids in discerning motivic variations from the main instrument. For most of a performance, this makes sense. However, at a certain point, the instrumentalist may cede the floor to the drummer. At this point, the instrumentalist is likely to repeat the same motif over and over, allowing the drum to engage in variations. Listeners familiar with Hindustani music will shift their attention to the drum, relegating the instrumentalist to the background, where his or her repetitions will help to frame the variations in the drum. However, other listeners will often fail to do this, thus producing a different response.

Even when listeners have the same foreground and background object, they may not have the same degree or kind of pattern recognition. For example, when I teach Hindustani music, I generally find that most students are sensitive to the pulse of the drumming without instruction. However, few if any are sensitive to its cyclic quality, the repetition of the drumming pattern after a fixed number of beats. Put differently, a few students could not even tap their foot to the beat of the music. Most could do this; however, they could not say when a rhythmic cycle began or how long it extended (seven beats? eight beats? twelve beats?). This is important because part of the aesthetic pleasure one experiences in listening to Hindustani classical music involves ways in which the rhythmic cycle creates, frustrates, and fulfills expectations for the resolution of melodic improvisations on the main instrument. One misses this entirely if one responds only to the pulse and not to the cycle.

Technically, this is a matter of "encoding." Encoding is the process whereby our mind selects elements of experience, segments them into units, and structures them into relations with one another. Encoding is a process that recurs at various levels of processing. For example, visual neurons are sensitive to only certain aspects of the environment. They fire only in the presence of certain phenomena-for instance, a line with a particular orientation in a particular area of the visual field (see Wurtz and Kandel 2000a, p. 534). Thus they select only that information. Through "lateral inhibition" (reducing the likelihood that neighboring neurons will fire [see TessierLavigne 2000, p. 521]), they enhance the salience of that line, thus making an object's edge clearer to perception. This is a form of structuring. At subsequent levels of processing, some configurations of lines are selected and further structured, producing object perception (see Wurtz and Kandel 2000b, pp. 564-565). In the case of Hindustani drumming, my students were not encoding the rhythmic cycles; their encoding stopped with the rhythmic pulse and did not extend to the cycle.

Encoding affects one's response to focal phenomena as well. First and most obviously, degree of encoding will affect one's pattern recognition. A simple case is variation on a theme. In Hindustani classical music, as in most if not all other traditions of music, a great deal of the aesthetic pleasure results from ornamentations, juxtapositions, and/or transformations of motifs. When the scales overlap with those used in Western music and the instrument is familiar (e.g., a flute), my students seem to find it easier to hear at least some of the motifs in the instrumental performance. However, if the scale is very different and/or the instrument is unfamiliar, this becomes more difficult. For example, perhaps disoriented by the strangeness of the scale (roughly, F, Gb, A, B, C, Db, E), they may be unable to discern the main motifs of rāga Lalit. In consequence, the only 
pattern they hear is very general - a single instrument playing with a drum and a drone-and is therefore likely to give rise to habituation (and boredom) swiftly. A related problem may occur when the scale is more accessible, but the instrument is unfamiliar (e.g., a vīnā) or the soloist uses unfamiliar techniques (such as ongoing oscillation around scale tones in vocal performance). In connection with this, my impression is that, at least initially, students find it difficult to encode the difference between part of a motif played by plucking different strings and the same notes played by gliding from one note to the next by stretching a single string. What strikes them as mere repetition (thus a potential source of habituation, thus boredom), strikes me as a variation.

Finally, it seems clear that we have not only different degrees of pattern recognition and disorientation, but also different degrees of sensitivity to enjoyment of pattern recognition (i.e., different degrees of reward system reactivity) and different degrees of aversion to disorientation. Moreover, there are variations in the precise timing of our pattern recognition, even in cases when that recognition is shared. Even very small differences in timing could make a great difference in the degree of pleasure or aversion. I may get the joke only a fraction of a second after you do, but that fraction of a second may be enough to make the joke not terribly funny for me and hilariously successful for you. Moreover, there is undoubtedly some difference in the degree to which aversive emotional responses linger. Perhaps my irritation at disorientation lasts longer than yours, inhibiting my subsequent pleasure at pattern recognition, thus dampening or even overcoming my enjoyment.

In short, it seems clear that the apparently universal aesthetic principle of nonhabituated pattern recognition entailing reward system involvement is not merely compatible with variety in aesthetic response. It actually predicts such variety. The same point holds for the second main cognitive process and the second emotion system that seem to produce aesthetic response.

\section{Aesthetic Response and Idiosyncrasy (II): Prototype Approximation and Attachment}

While some research points toward non-habitual pattern recognition as definitive of aesthetic pleasure, another set of studies points toward prototype approximation. The difference may be a matter of single versus distributed targets (e.g., faces versus decorative art, such as calligraphic ornamentation), with music counting as a paradigmatic distributed target. Another possibility is that it is a matter of differences in processing streams, where the prototype-approximation condition applies to the ventral or "what" pathway while the pattern-recognition condition applies to the dorsal or "where" and "how" pathway (on the pathways, see Wurtz and Kandel 2000b, p. 548; for a different account of the possible relevance of these pathways in aesthetics, see chapter eight of Ramachandran 2011). In any case, we seem to respond to different targets as aesthetically pleasing depending on their conformity to one of these criteria. 
Prototype approximation is the degree to which a given target is or is not similar to one's prototype of the relevant kind of object. A prototype is roughly an average case and it is often discussed as an average case (for a concise account of prototypes, see Rosch 2011; on prototypes and averaging, see Mcleod et al. 1998, p. 63). However, it seems clear that the "averaging" process here is complex. For example, diet foods would include a range of items, from low-fat chicken to butterless vegetables. Clearly, an actual average of these foods would have a certain number of calories. However, for many people, the most prototypical diet food is zero-calorie lettuce (see Kahneman and Miller 1986, p. 143). This suggests that there is not simply an averaging process, but some sort of weighted averaging. Thus our minds do not count all instances of a given kind equally. Rather, we seem to give greater weight to some instances over others. A number of factors may contribute to this weighting. In Understanding Nationalism (Hogan 2009), I isolated five factors that seem to govern the degree to which one identity category is more important than another. These were saliency, functionality, contrast, emotional force, and durability (the list is not intended to be complete). The same factors are likely to be operative here, given that both are a matter of categorization, with each factor having an effect on prototyping. For example, the function of a diet food is to reduce caloric intake. Thus the most highly functional diet food would be the one with the least calories. Emotional force seems particularly important. If one has wretched memories of choosing to snack on lettuce leaves rather than one's favorite dish of crispy, deep-fried pork fat, that will likely have more force in defining the prototype than memories of choosing chicken over beef for one's main course. Prototypes also differ from actual averages in that they may vary with context. As Kahneman and Miller point out, in the context "Manhattan apartment," the prototypical dog will be rather different from that in the context "a farm in Maine" (1986, p. 140). In keeping with this, in the context of "dessert," the prototypical diet food is not lettuce, but perhaps non-fat frozen yoghurt.

There also seems to be a difference between continuous and discrete domains for prototypes. In discrete domains, the prototype may be readjusted from a weighted average to a particular sub-category. That may be the case with lettuce, clearly a discrete sub-category of diet foods. In contrast, categories that govern relatively continuous domains may be closer to actual averages. For instance, at least on certain dimensions, a prototypical cup would probably be about average height and width, rather than a particular sub-category of cup.

Returning to aesthetics, we find considerable research linking prototypes-or, in some cases, averages-with beauty (see Hansen and Topolinski 2011, p. 710 and citations). The results are very robust, ranging from faces (Langlois and Roggman 1990) to colors (Martindale and Moore 1988, p. 670) to cars (Halberstadt and Rhodes 2003) to performances of sonatas (Repp 1997). There are two problems with some of this research, however. The first is that it often does not distinguish averages from prototypes. Thus the research on facial beauty by Langlois and Roggman (1990) stresses averages. However, Russell's (2003) work indicates that enhancing facial luminance differences between men and women enhances aesthetic appeal (see also Rhodes 2006). In other words, making the women's faces more 
"feminine" in facial luminance makes them more appealing, whereas the opposite is the case for men's faces. This points toward prototypes, with their weighting of contrasting features by category, rather than strict averages. The point is related to Ramachandran's observations on beauty. His example of Tamil statuary (2004, p. 42) provides a case in point, as does classical Greek sculpture. We may instance, for example, a Standing Parvati (from Tamil Nadu, India, during the Chola Period of the ninth to twelfth century C.E. [see http://commons.wikimedia.org/wiki/File:MET_ Standing_Parvati.jpg]) and Polykleitos's Spear Bearer (from Greece, fifth century $\mathrm{BCE}$ ). Anyone who has visited a beach or a locker room should recognize that these are not strict averages, but enhance distinctive female and male properties respectively.

In sum, we have good reason to posit prototype approximation as a universal principle guiding aesthetic response (i.e., a feeling of beauty; response to art is clearly much broader than a response to beauty). What does this tell us about variability in taste? That variability is not likely to be random or unconstrained, since our prototypes are unlikely to diverge too massively. They will be constrained by averaging (which, again, enters into prototypes) and by certain broad tendencies, such as enhancement of difference from contrasting categories, such as male or female. (On the last point, in addition to the works cited earlier, see also Chatterjee's discussion of Ramachandran on "peak shift" or the enhancement of response to distinguishing properties [Chatterjee 2014, pp. 45-47.]) Nonetheless, as with nonhabitual pattern isolation, the principle predicts considerable difference in particular aesthetic responses. Here, too, the nature of the universal is such that it in effect requires at least some diversity in its particularization.

It may seem that the result of tacit averaging processes should be the same across individuals. But our averaging processes are not self-conscious, statistically strict procedures. They are, rather, implicit, heuristic processes. Crucially, they do not begin with a random sample, but with individual memories. Even if all individual memories counted equally (which they do not), there would be significant differences across individuals' sets of memories. Consider the case of facial prototypes (the same points hold for all relevant prototypes - for example, prototypes relating to stories). First, we see different people (read or hear different stories, and so on). Second, and perhaps more importantly, we see people with different frequencies. It is possible that we average our observations across individuals. However, it seems more likely that we average our observations across instances-or, at least, that frequency and duration of experience affect the average. Suppose I see Jones ten times every day, Monday through Friday, sometimes meeting for long conversations on weekends. In contrast, I briefly passed Smith in the hallway. It is possible that Jones's face and Smith's face will each count equally in my implicit facial averaging and in the formation of a facial prototype, since each is one individual face. However, it seems far more likely that my implicit averaging will be an ongoing process, affected to some extent by each reappearance of Jones, but only by the single appearance of Smith - thus counting Jones much more than Smith. In short, the sets across which we are averaging are very different. 
Second, it is at least possible that there are critical period experiences in prototype formation. Critical periods are developmental stages when cognitive or affective systems are subject to particularly formative experiences. For example, experiences during the critical period for language acquisition largely determine the languages in which we will have native speaker fluency. We may learn other languages at later ages, but with much more effort and usually far less success. It is at least possible that something like this occurs in childhood with beauty-not that our beauty-defining prototypes are fixed forever in the first few years of life, but that early experiences may form an initial prototype or set of prototypes that have a great degree of influence on later prototype formations. In other words, early prototype formations may bias later averaging processes. This is particularly consequential as it seems likely that infantile experiences are, if anything, more idiosyncratic than adult experiences, given that infantile experiences tend to be more limited.

Third, writers in situated cognition have shown that, in actual practice, our interactions with the world do not simply involve fixed cognitive structures applied uniformly to experiences with the external world. Rather, our cognition is constantly changing and reforming itself in ongoing interaction with the world (see Robbins and Aydede 2009). Cognitive processing could hardly be different in aesthetic response. Thus our formation of prototypes does not occur once and for all. Each new experience alters our prototypes. Indeed, following Barsalou (2009, p. 244), we might infer that, to a great extent, our prototypes are formed ad hoc and in specific contexts, more strongly influenced by recent cases than would be the case if this were a matter of simple averaging. In terms of facial prototypes, this means that the context of faces we have recently seen is likely to alter our prototypes and thus our sense of just what approximates the relevant prototype. Here, as elsewhere, the point holds for a range of cases, not just facial beauty. Moreover, perhaps in contrast with Barsalou, I suspect that the effects of context may not only be shortterm, but medium-term as well. For example, a student or teacher's response to a particular literary work is in part dependent on what other works are being read in the class. Such effects would probably be a matter of what works are given particular activation in reading the new work. The extended context of the class as a whole would tend to make other works from the class more salient, thus more significant for averaging.

The mention of salience brings us to another set of output differences, those connected with weighting variables - again, beyond salience, these include durability, function, contrast, and emotional force. Durability is the most equivocal. The idea here is that objects involve properties and conditions. Our minds abstract from the changeable conditions, isolating what is durable. To take a simple example, different directions and sources of light will affect a face-for example, what parts are illuminated and what are shadowed. In forming an image of the face, our minds in some degree subtract the lighting effects (if they did not, we would find it difficult to recognize someone in different lighting conditions). This process should repeat itself across faces, leading to prototypes that bear on enduring features of faces rather than ephemera. As far as I can tell, people generally abstract from circumstances in much the same way, so this is largely irrelevant to the issue of diversity in output. Indeed, 
the most interesting issues here concern why we find certain sorts of lighting or other ephemera more aesthetically pleasing. This could be because the situations are more prototypical or, what is perhaps more likely, that they make the target appear more prototypical.

Function is of somewhat limited value here as well. Responses to functionality appear, in general, fairly uniform. Perhaps the main differences involve professional or related specialization. Literary critics will view some features of a work as functional in that they will contribute to teaching or research; authors will encode some aspects as functional in inspiring their own creativity, or inhibiting it. These functional emphases are likely to lead to certain features of works and certain types of work entering significantly into the prototype formation of professionals in a way that they do not enter into the prototype formation of non-professionals. This is important because it suggests one reason why it is likely that there will be a difference between professional and non-professional tastes. (Other reasons include different sorts of habituation and different skills at encoding and pattern recognition in a particular area. Some of the complexity of the issue is brought out by Nodine et al. (1993). Though they formulate their findings in different terms, their research may suggest that there are differences in preferred type of aesthetic process, with art-trained viewers of paintings stressing pattern-recognition across figures rather than prototype-approximation of figures.)

The point bears most obviously on complex works of art. However, it extends well beyond that. An amusing example concerns a lab technician's observation, after a colonoscopy, that a relative had a "cute colon." She showed me the photograph, to which I actually had a mild disgust reaction, and certainly did not experience aesthetic pleasure. Presumably what was going on was that the lab technician had seen so many colon pictures that she had formed a prototype. In addition, the lab technician's assessment of the aesthetics of internal organs was also in part a matter of contrast. She not only had experience of colons generally, she had experience of healthy and unhealthy colons. Indeed, her categorization here was presumably not simply "colon," but "healthy colon" versus "unhealthy colon." The features of a healthy colon were weighted due to their contrast with unhealthy colon features. The reader will no doubt be delighted (if also moderately repulsed) to learn that the relative in question had a very healthy colon indeed. This contributed to the laboratory technician's aesthetic evaluation. When profession-related differences occur, they may be highly significant, as in this case. However, they remain a somewhat limited case.

As this example suggests, contrast is inseparable from categorization. The point holds outside medicine, as indicated by Russell's research on male and female faces (Russell 2003). Indeed, categorization and contrast are among the most important variables in aesthetic response. If one's aesthetic response to a target is in part a matter of prototype approximation, then clearly categorization is crucial even independent of contrast, because categorization determines just what prototype is activated. If you and I categorize a target differently, we are likely to have different aesthetic responses. The point is suggested by a popular Hans Christian Andersen story, "The Ugly Duckling" ("Den grimme Ælling” [Andersen 1949]). In that story, 
a poor, orphaned waterfowl is thought to be quite unaesthetic, until he is recognized as a swan, rather than a duck. One way of understanding the story is in terms of categorization and prototype approximation. "Duck" and "swan" activate different prototypes. While the "ugly duckling" did not closely approximate a prototypical duck, he did closely approximate a prototypical swan.

The point is not confined to commonly shared categories, such as duck and swan. Indeed, it applies perhaps most significantly to more or less fine-grained subcategories, including professional subcategories. Research on emotion categorization has shown that the more or less fine-grained quality of our emotion categorizations affects the experience and course of those emotions (see Lindquist and Barrett 2008). We may expect the same sort of consequences for categorization and subcategorization elsewhere. For example, when Northern Europeans first saw sub-Saharan Africans, they had no option but to categorize their faces in the same way that they categorized their own faces. Since there were highly salient ways in which Africans deviated from the statistical average for Europeans, they were very likely to find Africans ugly, at least in those respects. The same point holds for subSaharan Africans seeing Europeans. This would change either when the unusual group (whether Africans or Europeans) began to affect prototype formation on the part of the home society or when members of the home society formed subcategories with associated prototypes, such that they began implicitly or explicitly to judge a "beautiful European face," a "beautiful African face," and so on. In keeping with this, research indicates that "ratings of facial attractiveness" are consistent "across ethnicities and cultures" (Chatterjee 2014, p. 7) when the groups involved are not isolated from one another. In contrast, the limited contemporary research on the topic indicates that, when the groups are isolated and there are differences in appearance, then aesthetic response differs (see Chatterjee 2014, pp. 7-8 and citations). This contemporary research is of course supported by historical materials related to colonialism and other early contact (e.g., the association of black skin with ugliness in Shakespeare's Othello, as in the characterization of Othello as a "sooty [... ] thing" who gives rise "to fear, not to delight" [Shakespeare 1986, I.ii.69-70]).

The importance of categorization and contrast extends beyond faces, encompassing for example artistic movements and literary genres. Categorizing a particular work as "a painting" or even "a painting of the Madonna and child" may produce a very different effect than categorizing it as "early Mannerist," since the relevant prototypes would differ, as would the ways we encode the features of the work itself (the importance of categorization is stressed particularly by Carroll [2009], in a different theoretical context). Indeed, categorization generally affects both prototypes and the encoding of the target. Consider an example from narrative. The Indian film Fanaa concerns a Kashmiri revolutionary and a young, blind Kashmiri woman. They meet, apparently fall in love, and culminate their relationship. The revolutionary then leaves the woman and rejoins the revolution (after securing medical care to restore her sight). The ending of the film reunites them briefly, before the woman has to kill the revolutionary to prevent a possible terrorist attack. Though the film has many serious flaws, I probably find it much more aesthetically successful than would many other viewers, largely because I categorize it as a seduction plot. (The seduction plot 
is a cross-cultural genre wherein a man seduces and abandons a woman, who often pursues him, with one or both often dying. On this genre, see Hogan 2011a, pp. 210-220.) Without this categorization and associated prototype, viewers are more likely to see the film simply as a loosely romantic melodrama. (I should perhaps note that, in this case, my response is complex. It combines prototype approximation and non-habituated pattern recognition, which is probably common in our response to works of art.)

The last variable that affects averaging is affective force. We would expect prototype formation to be disproportionately guided by strongly emotional instances, as opposed to neutral instances. It is well established that emotionally consequential instances tend to overwhelm statistical information in judgment tasks (see Nisbett and Ross 1980, p. 15). The formation of prototypes would seem to involve processes of the same general sort. In the case of aesthetic response, we may expect another emotional factor as well. The research we have been considering often treats prototype approximation alone. But presumably prototype approximation fosters a feeling of beauty only when it is connected with the right sorts of emotion. For example, prototypes related to disgust-provoking objects should not produce aesthetic delight. This may seem contradicted by the laboratory technician's comment about the colon picture. However, once again, she presumably subcategorized colons into "healthy" and "diseased." It seems highly unlikely that she would have found beauty (or "cuteness") in a picture of a colon showing a high degree of disease prototypicality.

In the case of the colon photograph, the emotions at issue are presumably joy and relief. The context of such an evaluation is rather like that of a story where a possible tragedy is reversed in a comic conclusion. The difference is that the fate of the protagonist in the technician's story is real and not merely simulated. This example suggests that aesthetic pleasure may arise with prototype approximation involving positive emotions generally, perhaps due simply to reward involvement.

However, there is some evidence that one sort of positive emotion is perhaps particularly significant for aesthetic feeling. That emotion is attachment, the sort of bonding one feels in parent/child relations, companionate love, and as one component of romantic love. (On the evidence here, see Hogan 2014a, b and Koelsch 2013, pp. 293-295.) If indeed attachment is especially important in fostering a feeling of beauty, then we would expect prototypes with a strong attachment component to be particularly germane to aesthetic response. More exactly, we would expect to find that the relevant prototypes stress attachment-based memories in averaging. These memories may concern literary works to which we feel attachment, places or characters to which we feel attachment, or real people to whom we feel attachment. In the case of facial beauty, for example, it would seem that our prototypes bearing on aesthetic feeling should stress the faces of attachment figures. Here we have another source of individual output variability, since our attachment figures differ greatly.

In my view, the attachment variable is likely to be the most important. Some authors appear to agree. I have argued elsewhere that in Mrs. Dalloway, Woolf presents an implicit account of beauty as largely attachment based (Hogan 2013). 
Sappho wrote that people say many different things are beautiful, but her claim is for "whatever one loves" (Barnard 1958, p. 41). Shakespeare suggests the point in sonnet 130_-"My mistress' eyes are nothing like the sun [...] And yet, by heaven, I think my love as rare/As any she belied with false compare" (in Bevington 1992, p. 1643). Taking up a broader, cultural source, Khan recounts that, when the legendary Arabic lover, Majnu, was asked about how he could be entranced by Layla's beauty since, in fact, she was "rather plain," he responded, "My Layla must be seen with my eyes" (1997, p. xxi). In each case, the point seems to be that a sense of beauty is bound up with attachment, at least in particular cases. The present analysis adds to this observation the idea that a history of attachment bonds might actually affect the prototypes through which one responds to targets as beautiful or not beautiful.

When I first began formulating these ideas, I was struck by the degree to which they explained my judgments about faces. Beyond faces, my sense of literary beauty seems to be affected by books that I "love"; cinematic beauty, by films that I "love," and so on. Sticking to the case of faces for the moment, it seems clear that my aesthetic response to Tanuja is in part the result of my attachment relations to my wife over the course of the past three decades. Lalita (see http://www.koausa.org/ Books/Sukeshi/index.html) is clearly more similar to Tanuja than to Miley Cyrus. Indeed, the effect of my attachment relation here is made even clearer if I contrast Tanuja with a woman that I considered strikingly beautiful when I was 15, Joey Heatherton. In looking at these faces, I am struck by the degree to which the young Heatherton resembles Cyrus, suggesting that my aesthetic response at 15 was much closer to that of Maxim Magazine than after 30 years of attachment bonding.

The case of my wife indicates that prototypes for aesthetic response may be altered in adulthood. However, the case with my male prototype may be different. Here, the obvious connection is with my father (see Fig. 1), my primary attachment object in childhood.

(Though not shown in this photograph, for many years my father even had pencil moustache, making his appearance even more similar to that of Raj Kapoor. I should note that I picked Tanuja and Raj Kapoor as good examples of my aesthetic response before I thought of explaining my aesthetic preferences in terms of attachment bonds.) This may point to the importance of critical period experiences. Indeed,

Fig. 1 The author (age 23 months) and his father

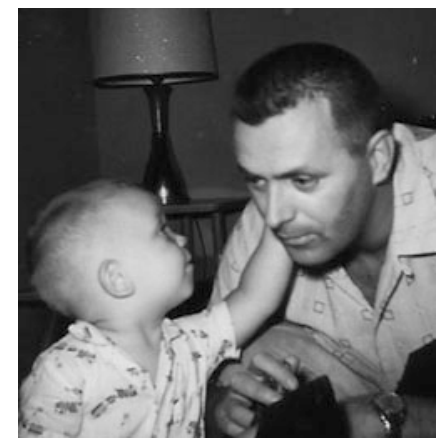


as my primary attachment figure, my father may have contributed to my larger prototype of a beautiful face. It is striking to me that the putatively most beautiful man and woman, Josh Hartnett and Miley Cyrus, actually look somewhat alike. In contrast, my own choices for aesthetic preference-Tanuja and Raj Kapoor-are somewhat similar as well. This suggests that people may have a broader, nongendered facial prototype that is to some extent particularized and differentiated into gender-specific prototypes in contrastive sub-categorization. If so, then it may be that my critical period experiences facilitated the adult change in my aesthetic prototype. Put simply, my wife's face exerted a greater effect on my facial averaging in part because its effects were not entirely dissimilar to those of my father's face-a striking case of complex, variant responses developing through universal principles.

Of course, it is difficult to say just how critical period experiences bear on works of art, as opposed to faces. It is possible that such experiences affect some domains of aesthetic response and not others. However, critical period experiences are clearly central to attachment. In consequence, if attachment is indeed crucial for a feeling of beauty, it seems likely that critical period experiences are important for our aesthetic response to works of art, at least in this respect. To take, once again, a personal example — some of my most intense aesthetic experiences bear on mystical literature, such as the poems of Rumi. Moreover, I seem to have a particular preference for mystical poetry relating to female divinity, prominently including many Bengali Goddess poems (see McDermott et al. 2001), which stand (perhaps with Rumi) as a paradigm of aesthetic excellence for me. This is to some degree surprising, since I am not religious. However, my earliest aesthetic experiences were bound up with Catholicism, and particularly to my family's Marianism, with its specific devotion to Our Lady of Częstochowa, or the "Black Madonna." It seems likely that these critical period experiences are affecting my current aesthetic preferences in literature and art.

\section{Conclusion}

In sum, it may seem at first that any assertion of aesthetic universals is undermined by the diversity of taste. However, this apparent contradiction dissolves when we examine the issue more carefully. First, we must distinguish between objective or essential beauty, social norms for beauty, and the psychology of aesthetic response. We set aside the first usage, since it is difficult to make sense of the idea of essential beauty as something parallel to, say, the chemical composition of water. The second and third meanings require us to make a further distinction, that between principles and the output of principles. In physics, the laws of nature produce different results when applied in different conditions. The same point holds for social norms of beauty and principles of the psychology of beauty. In both cases, we might give universal accounts of beauty without thereby requiring that the observable outcomes of the universals be uniform. 
There is evidence pointing toward two important cognitive contributors to aesthetic response: non-habituated pattern recognition and prototype approximation. There also appear to be two particularly important motivational systems: endogenous reward and attachment. If the principles governing aesthetic response are indeed governed largely by these four factors, our universals actually predict that there will be considerable diversity in outputs, which is to say in feelings of beauty. Among other things, our encoding of targets thus our ability to isolate patterns will vary, as will our proneness to habituation and our reward system sensitivity. Our categorizations will differ, often in terms of specificity, thus changing what prototypes are activated for a particular target. Our actual prototypes will be different due to individual variety of experience, context, emotional variables, and other factors. In short, the currently most plausible universals of beauty not only do not require uniformity of output; they are not even compatible with uniformity of output.

Open Access This chapter is distributed under the terms of the Creative Commons Attribution Noncommercial License, which permits any noncommercial use, distribution, and reproduction in any medium, provided the original author(s) and source are credited.

\section{References}

Andersen, Hans Christian. 1949. The Ugly Duckling. Trans Jean Hersholt. http://www.andersen. sdu.dk/vaerk/hersholt/TheUglyDuckling_e.html.

Barnard, Mary, ed. and trans. 1958. Sappho: A new translation. Berkeley: University of California Press.

Barsalou, Lawrence. 2009. Situating concepts. In The Cambridge handbook of situated cognition, ed. Philip Robbins and Murat Aydede, 236-263. Cambridge: Cambridge University Press.

Bevington, David (ed.). 1992. The complete works of Shakespeare, 4th ed. New York: Harper Collins.

Carroll, Noël. 2009. On criticism. New York: Routledge.

Chatterjee, Anjan. 2014. The aesthetic brain. Oxford: Oxford University Press.

Eagleton, Terry. 1990. The ideology of the aesthetic. Oxford: Basil Blackwell.

Eriksen, Jens-Martin, and Frederik Stjernfelt. 2012. The democratic contradictions of multiculturalism. New York: Telos Press.

Faludi, Susan. 1991. Backlash: The undeclared war against American women. New York: Crown.

Fareri, Dominic, and Mauricio Delgado. 2013. Reward learning: Contributions of corticobasal ganglia circuits to reward value signals. In The Cambridge handbook of human affective neuroscience, ed. Jorge Armony and Patrik Vuilleumier, 444-464. Cambridge: Cambridge University Press.

Giles, David. 2010. Psychology of the media. New York: Palgrave Macmillan.

Halberstadt, Jamin, and Gillian Rhodes. 2003. It's not just average faces that are attractive: Computer-manipulated averageness makes birds, fish, and automobiles attractive. Psychonomic Bulletin and Review 10: 149-156.

Hansen, Jochim, and Sascha Topolinski. 2011. An exploratory mindset reduces preference for prototypes and increases preference for novel exemplars. Cognition and Emotion 25: 709-716.

Hogan, Patrick. 2003. The mind and its stories: Narrative universals and human emotion. Cambridge: Cambridge University Press. 
Hogan, Patrick. 2009. Understanding nationalism: On narrative, cognitive science, and identity. Columbus: Ohio State University Press.

Hogan, Patrick. 2011a. Affective narratology: The emotional structure of stories. Lincoln: University of Nebraska Press.

Hogan, Patrick. 2011b. What literature teaches us about emotion. Cambridge: Cambridge University Press.

Hogan, Patrick. 2013. Literary aesthetics: Beauty, the brain, and Mrs. Dalloway. In Literature, neurology, and neuroscience: History and modern perspectives, ed. Stanley Finger, Anne Stiles, and François Boller, 319-337. Oxford: Elsevier.

Hogan, Patrick. 2014a. Stylistics, emotion and neuroscience. In The Routledge handbook of stylistics, ed. Michael Burke, 516-530. New York: Routledge.

Hogan, Patrick. 2014b. Attachment system involvement in esthetic response. Archives of Neuroscience 1(3): e18173.

Kahneman, Daniel, and Dale Miller. 1986. Norm theory: Comparing reality to its alternatives. Psychological Review 93: 136-153.

Khan, Zia Inayat. 1997. Preface to the 1997 edition. In The story of Layla and Majnun, ed. Nizami. Rudolph Gelpke, English version in collaboration with E. Mattin and G. Hill, final chapter trans. Zia Inayat Khan and Omid Safi, xix-xxi. New Lebanon: Omega Publications.

Koelsch, Stefan. 2013. Emotion and music. In The Cambridge handbook of human affective neuroscience, ed. Jorge Armony and Patrik Vuilleumier, 286-303. Cambridge: Cambridge University Press.

Langlois, Judith, and Lori Roggman. 1990. Attractive faces are only average. Psychological Science 1: 115-121.

Lindquist, Kristen, and Lisa Feldman Barrett. 2008. Emotional complexity. In Handbook of emotions, ed. Michael Lewis, Jeannette M. Haviland-Jones, and Lisa Feldman Barrett, 513-530. New York: Guilford Press.

Macaulay, Thomas Babington. 1835. Minute by the Hon'ble T. B. Macaulay, dated the 2nd February 1835. Minute on Education. Available at http://www.columbia.edu/itc/mealac/pritchett/ 00generallinks/macaulay/txt_minute_education_1835.html.

Martindale, Colin, and Kathleen Moore. 1988. Priming, prototypicality, and preference. Journal of Experimental Psychology. Human Perception and Performance 14: 661-670.

McDermott, Rachel Fell, ed. and trans. 2001. Singing to the goddess: Poems to Kālī and Umā from Bengal. Oxford: Oxford University Press.

McLeod, Peter, Kim Plunkett, and Edmund Rolls. 1998. Introduction to connectionist modelling of cognitive processes. Oxford: Oxford University Press.

Nisbett, Richard, and Lee Ross. 1980. Human inference: Strategies and shortcomings of social judgment. Englewood Cliffs: Prentice-Hall.

Nodine, Calvin F., Paul J. Locher, and Elizabeth A. Krupinski. 1993. The role of formal training on perception and aesthetic judgment of art compositions. Leonardo 26: 219-227.

Ortony, Andrew, Gerald L. Clore, and Allan Collins. 1988. The cognitive structure of emotions. Cambridge: Cambridge University Press.

Pandit, Lalita. 1995. Caste, race, and nation: History and dialectic in Rabindranath Tagore's Gora. In Literary India: Comparative studies in aesthetics, colonialism, and culture, ed. Patrick Hogan and Lalita Pandit. Albany: State University of New York Press.

Ramachandran, Vilayanur S. 2004. A brief tour of human consciousness. New York: Pi Press.

Ramachandran, Vilayanur S. 2011. The tell-tale brain: A neuroscientist's quest for what makes us human. New York: W. W. Norton.

Repp, Bruno. 1997. The aesthetic quality of a quantitatively average music performance: Two preliminary experiments. Music Perception 14: 419-444.

Rhodes, Gillian. 2006. The evolutionary psychology of facial beauty. Annual Review of Psychology 57: 199-226.

Robbins, Philip, and Murat Aydede. 2009. A short primer on situated cognition. In The Cambridge handbook of situated cognition, ed. Philip Robbins and Murat Aydede, 3-10. Cambridge: Cambridge University Press. 
Rosch, Eleanor. 2011. Prototypes. In The Cambridge encyclopedia of the language sciences, ed. Patrick Hogan, 680-682. Cambridge: Cambridge University Press.

Russell, Richard. 2003. Sex, beauty, and the relative luminance of facial features. Perception 32: 1093-1107.

Said, Edward W. 1978. Orientalism. New York: Pantheon.

Said, Edward W. 1993. Culture and imperialism. New York: Alfred Knopf.

Shakespeare, William. 1986. The tragedy of Othello the Moor of Venice, ed. Alvin B. Kernan. New York: Signet.

Tessier-Lavigne, Marc. 2000. Visual processing by the retina. In Principles of neural science, 4th ed, ed. Eric Kandel, James Schwartz, and Thomas Jessell, 507-522. New York: McGraw-Hill.

Vuust, Peter, and Morten L. Kringelbach. 2010. The pleasure of music. In Pleasures of the brain, ed. Morten L. Kringelbach and Kent Berridge, 255-269. Oxford: Oxford University Press.

Watt, Ian. 1957. The rise of the novel. Berkeley: University of California Press.

Williams, Raymond. 1985. Keywords: A vocabulary of culture and society, Revised ed. Oxford: Oxford University Press.

Wurtz, Robert H., and Eric Kandel. 2000a. Central visual pathways. In Principles of neural science, 4th ed, ed. Eric Kandel, James Schwartz, and Thomas Jessell, 523-547. New York: McGrawHill.

Wurtz, Robert H., and Eric Kandel. 2000b. Perception of motion, depth, and form. In Principles of neural science, 4th ed, ed. Eric Kandel, James Schwartz, and Thomas Jessell, 548-571. New York: McGraw-Hill.

Zebrowitz, Leslie, Joann M. Montepare, and Hoon Koo Lee. 1993. They don't all look alike: Individuated impressions of other racial groups. Journal of Personality and Social Psychology 65: 85-101. 